\title{
Does Safety Netting for Lung Cancer Symptoms Help Patients to Reconsult Appropriately? A Qualitative Study
}

Georgia Black ( $\sim$ g.black@ucl.ac.uk)

University College London

Sandra van Os

University College London

Christina Renzi

University College London

Fiona Walter

Queen Mary University of London

Willie Hamilton

University of Exeter

Katriina L Whitaker

University of Surrey

\section{Research Article}

Keywords: Lung cancer, early diagnosis, primary care, safety netting, diagnostic uncertainty

Posted Date: September 27th, 2021

DOl: https://doi.org/10.21203/rs.3.rs-908030/v1

License: (c) (i) This work is licensed under a Creative Commons Attribution 4.0 International License. Read Full License 


\section{Abstract \\ Background}

Safety netting in primary care is considered an important intervention for managing diagnostic uncertainty. This is the first study to examine how patients understand and interpret safety netting advice around low-risk potential lung cancer symptoms, and how this affects reconsultation behaviours.

\section{Methods}

Qualitative dyadic interview study in UK primary care. Pre-covid-19, five patients were interviewed face-toface twice (shortly after a primary care consultation for potential lung cancer symptom(s) and 2-5 months later). The general practitioner (GP) they last saw was interviewed face-to-face once. During the covid-19 pandemic, an additional 15 patients were interviewed once via telephone. Audio-recorded interviews were transcribed verbatim and analysed using a mix of inductive and deductive thematic analysis.

\section{Results}

The findings from our thematic analysis suggest that patients prefer active safety, as part of thorough and logical diagnostic uncertainty management. Passive safety netting may be perceived as dismissive and cause delayed reconsultation. GP safety netting strategies are not always understood, potentially causing patient worry and dissatisfaction. Telephone consultations and the diagnostic overshadowing of COVID-19 on respiratory symptoms impacted GPs' safety netting strategies and patients' appetite for active follow up measures.

\section{Conclusions}

Safety netting guidelines do not yet offer solutions that have been proven to promote symptom vigilance and timely reconsultation for low-risk lung cancer symptoms. Patients prefer active safety netting coupled with thorough consultation techniques and a comprehensible diagnostic strategy, and may respond adversely to passive safety netting advice.

\section{Background}

Safety netting is considered an important intervention for managing low-risk symptoms of cancer $(1,2)$, particularly in the UK where it forms part of the government guideline for suspected cancer management(3). Safety netting refers to actions taken and advice given to patients by healthcare practitioners about how to monitor and re-seek help for new, recurrent, persistent, or worsening symptoms, which may benefit patients in terms of disease stage at diagnosis, treatment options and 
survival(4). Safety netting is a high-volume activity, with GPs reporting in a qualitative study that they use some form of safety netting at the end of almost every consultation(5). Indeed, an audit of patient records showed that safety netting is recorded in $44 \%$ of all patient contacts where a cancer is eventually diagnosed(6). Safety netting is particularly important in the diagnostic management of lung cancer, where low predictive value symptoms such as cough and tiredness are the most common first complaints that patients present to primary care with $(7,8)$. Safety netting is transactional(5), and is not effective if the patient does not hear or understand the advice they are given by the primary care healthcare professional (HCP) or if the advice is insufficiently specific $(9,10)$. Heyhoe et al.(11) suggest that, as part of effective safety netting, HCPs and patients should work together to develop and agree strategies that encourage sharing of symptom monitoring, re-appraisal and feedback, which will aid diagnosis of cancer at an early stage(12).

Poorly communicated safety netting advice may be worse than none; patients may delay reconsultation for lung symptoms by several months if they perceive that symptoms have been initially attributed to a benign cause, for example chronic obstructive pulmonary disease(13-16).

Best practice guidelines for safety netting have been developed through consensus(17) and evidence synthesis(10), although HCPs may deviate from this in practice $(1,18)$. There is some evidence to suggest that patients prefer clear directives, for example setting a specific timescale for reconsultation rather than an open-ended invitation(19); however, there is no evidence about whether patients understand or attend to safety netting advice, nor how this affects reconsultation behaviours.

This study used a qualitative dyadic (paired) design to understand how patients responded to safety netting in primary care for low predictive value symptoms related to lung cancer. We aimed to capture the effect of safety netting on the way patients judge their symptoms and (re)seek help in primary care over time.

\section{Methods}

\section{Recruitment}

Three GPs and 20 patients (Table 1) were recruited purposively to achieve variation in age, gender, geographic spread and ethnic background. The first five patient participants were recruited in general practices in deprived areas (lowest $30 \%$ SES) by local National Institute for Health Research Clinical Research Network research nurses. Patients meeting inclusion criteria were given a study recruitment pack and encouraged to contact the researcher if interested in participating. Once patients were recruited, the GP they saw most recently was also approached for interview. Participants gave written consent before interview.

Due to COVID-19, the recruitment strategy was amended in March 2020. A specialist recruitment agency, Taylor McKenzie, recruited 15 participants using a database of potential research participants and by approaching patient support groups. A screening questionnaire ensured participants had recently 
presented to their GP with a low predictive value symptom related to lung cancer and were also from the lowest $30 \%$ SES. Verbal informed consent was audio recorded.

Table 1: Participant demographics and interview characteristics

\section{Patient characteristics}

Gender

Female

Age

Ethnicity

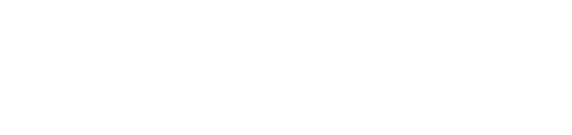

\begin{tabular}{|c|c|c|}
\hline \multirow[t]{2}{*}{ Gender } & Female & 13 \\
\hline & Male & 7 \\
\hline \multirow[t]{2}{*}{ Age } & Range & $40-69$ \\
\hline & Mean & 50.9 \\
\hline \multirow[t]{3}{*}{ Ethnicity } & White & 13 \\
\hline & Black & 6 \\
\hline & Asian & 1 \\
\hline \multirow[t]{8}{*}{ Presenting problems (some participants reported more than 1) } & Fatigue & 14 \\
\hline & Cough & 11 \\
\hline & Shortness of breath & 6 \\
\hline & Appetite loss & 2 \\
\hline & Anaemia & 2 \\
\hline & Chest pain & 1 \\
\hline & Weight loss & 1 \\
\hline & Difficulty sleeping & 1 \\
\hline \multirow[t]{4}{*}{ Deprivation score by postcode } & Most deprived 5\% & 2 \\
\hline & Most deprived 10\% & 8 \\
\hline & Most deprived $20 \%$ & 6 \\
\hline & Most deprived 30\% & 4 \\
\hline
\end{tabular}

Male

7

Range

40-69

Mean

50.9

White

13

Black

Asian

6

\section{GP characteristics}

Gender

Female

2

Male 
The first five participants were interviewed face-to-face twice in a private room at the practice, once shortly after the consultation and again 2-5 months later. Their GPs were interviewed once face-to-face in their consultation room. The remaining 15 patient participants were interviewed once via telephone. Before starting the interview, the interviewer made sure that the participant had recently presented to their GP with a potential lung cancer symptom. Flexible interview topic guides (appendix 1) were developed using published literature and feedback from patient representatives. All interviews were conducted by an experienced, female, qualitative social science researcher. Interviews were digitally recorded and transcribed verbatim.

\section{Analysis}

Transcripts were imported into NVivo 11, qualitative data analysis software, and coded by two authors using an inductive approach. Author 1 and 2 (initials removed to allow blind review) initially read all transcripts, and author 1 coded all transcripts with a particular focus on GP-patient dyads. To ensure the coding represented the data it was discussed with author 2. After analysis of the five dyads, the remaining dataset of 15 single patient transcripts was coded. Initial codes were then grouped into potential themes, and a thematic map was produced. The themes were then discussed and finalised by author 1 and 2, who agreed that the final themes were supported by the data. The final analysis and a draft of this manuscript were checked by all co-authors.

\section{Results}

\section{Theme 1: Patients prefer active safety netting strategies}

Patients preferred safety netting that included advice and actions that actively promoted re-consultation or involved pro-active follow-up.

[l appreciated the] information in the initial conversation with the receptionist, and the prompt contact from the doctor. And the advice, and him wanting to know, in a few days how I was feeling, was anything getting any worse, any more symptoms. (Patient 20, female, 57, telephone interview).

Active safety netting as part of a thorough and logical approach to managing their lung cancer-relevant symptoms allowed patients to understand the diagnostic strategy, in turn making the safety netting advice easier to understand. For example, in the example presented in Box 1 both the patient and GP describe the 'logical'steps the GP took to reach the diagnosis over several visits. In his first interview the patient described how the GP had addressed each of his concerns through a series of logical steps, in his second interview he reflected on how this approach had been reassuring.

Box 1: Thoroughness in consultation (Patient 4 and GP 2) 
Patient 4, male 47 years old

Most important thing was she was able to listen. She listened to me when I said my symptoms were and then she talked me through, saying, "I've listened to your chest at the front. Your chest is clear. There's nothing to see in your throat. Your nose is not blocked. So, let's get these tests done." So, the first consultation, "Let's do these tests and make sure everything's alright, blah, blah, blah." Everything that I said she was able to check it and say, "This is this, this is this, this is this." Then okay, if it wasn't showing up as a regular cold and she couldn't identify a virus as yet, "let's do these tests to make sure it's nothing else." She also spoke to somebody on the "phone, not within the practice, but I think it was at [hospital] and confirmed things. So, it felt like everything she was doing was logical and that was...yeah, I was reassured. Especially when the tests came back and there was nothing untoward, so that was a relief.

\section{GP2, female}

I think at the beginning he came in; he'd had a cough; I think he'd had some recent travel and I thought it was a chest infection hence we went for the antibiotic route. I think he then saw one of my colleagues, had a similar story, treatment and then it was this persistent cough at which point I was thinking oh what's going on. Is this something else or is it just an allergic cough? I think that's why I gave the steroids. I'm relieved to know that I'm true because I couldn't ...he was otherwise okay. He hadn't lost weight; he hadn't lost his appetite. He was otherwise stable, and I don't think he's a smoker, so I couldn't. He was quite an unclear one because I couldn't. From a cancer side yes, you'd be thinking he's got a persistent cough and I think I did request a chest X-ray as well. So that was my cover but [...] I think he was worried, and he was suffering more than anything. I don't know, but I think it was more that he was getting frustrated that his cough wasn't settling. I'm not sure we had any magic cures for it and that's why he was coming back.

Several patients commented that their telephone consultation was with a GP they had not met before, who had not read their notes and was unaware of their history. They were dissatisfied with the level of thoroughness in the consultation, were not sure that all possible diagnoses had been considered, and felt 'unsafe' as a result.

If I get on the phone and talk to a GP, and they work through my symptomology, I discuss some of my background, what's been happening with me, get some historical data, and to then come to a conclusion in terms of a prognosis. Had that been done, in that kind of environment, I suppose, I would have thought "You know what, they've taken the time to research what's going on with, happen with me historically". (Patient 14, male, 50, telephone interview)

Patients who had attended a telephone consultation during the COVID-19 pandemic, where the focus was perhaps mostly on acute symptoms and/or ruling out COVID-19, found it difficult to remember everything they wanted to discuss with the GP without a thorough discussion of wider issues.

GPs reported that they used active safety netting with certain patients, but not others, particularly when their "level of worry is a bit higher" (GP 3). Two GPs were concerned that active safety netting could worry patients unnecessarily.

A lot of what we do in general practice is actually reassure the worried well and a lot of discussions about cancer safety netting is actually just doing the exact opposite. (GP 1, male). 
GP 3 believed that explicitly naming red flag symptoms could raise anxiety among patients at very low risk of cancer.

Once you explain the list of things to look out for that people start getting them more often. [...] I don't think it's enough of a negative not to do it. But certainly, there are a few patients where almost anything, if you'd asked them about it, they'd, manage to find an example of it. (GP3, female).

This suggests that GPs have particular concerns about specific safety netting devices, including highlighting red flag symptoms, for fear of generating anxiety, although this was not reported in our patient interviews. Patients engaged with diagnostic uncertainty and management as part of a thorough and attentive consultation, especially when active steps were taken to promote re-consultation.

\section{Theme 2: Patients respond poorly to passive safety netting strategies}

Patients interpreted passive safety netting (verbal instructions to come/call back if symptoms do not resolve) as dismissive, and a sign that the GP was uninterested in their problem. This was a particularly salient experience for patients who had a telephone consultation. For example, Patient $13 \mathrm{had}$ a telephone consultation during the pandemic, and inferred that the GP was not "that interested", despite their assurances that she should get in contact if her symptoms persisted:

I think I had the cough and the fatigue for about a week. And so that's when I rang up the doctor, and the doctor weren't really that interested. Told me to ring back if I got any worse. He was more concerned about my chest, but my chest was fine. I think he was going on the lines of this virus rather than anything else...yeah. Get in contact if it gets worse, or if it is serious phone the NHS, that was it! I felt a bit daft when I've come off the phone [...] I wasn't offered anything. Not at all. (Patient 13, female, 48, telephone interview)

Some patients experienced feelings of shame following passive safety netting, and felt dismissed particularly when access to a blood test was restricted. These patients did not receive specific advice on how to deal with COVID-related disruptions to additional tests they may need.

And so, there is nothing they suggest 'Oh, call me back at such and such at a later time and we'll check it. Obviously, the problems is right now I can't go and get a blood test. So, I guess that's made it problematic. Or a follow-up appointment. [...] Yeah, it did feel rushed. This is what you're getting, and see you later. (Patient 10, female, 40, telephone interview)

GPs justified passive safety netting in consideration/management of cancer risk, particularly in face to face consultations where they felt more confident about their appraisal of the patient's likelihood of cancer. 


\section{Theme 3: Patients don't always know about diagnostic strategy, either because they don't understand it or where GP hasn't told them everything}

GPs described diagnostic strategies predominantly in relation to managing cancer risk, including some strategies not communicated to patients, such as heuristics about patient's characteristics (e.g. age), and future plans in the event that symptoms persisted. When patients were unaware or had not understood the GPs 'logic' or diagnostic strategy, there was often a feeling of concern or lack of resolution. Box 2 presents an example of misalignment between patient and GP, where the patient was unaware that the GP was ruling out anaemia [to exclude cancer] and did not feel reassured by blood tests and an x-ray as a diagnostic management strategy. She was, however, reassured following an MRI scan, which she felt was the only way to properly rule out cancer.

Box 2: Diagnostic strategy did not reassure patient (Patient 1 and GP 2)

\section{Patient 1 (female, 62 years old)}

But he said there is nothing seriously going on, it's just your nerve endings are a bit, sort of, getting old, I suppose, and we all get aches and pains as we age. So, I'm happy with that, because at least I know they've had a good look inside me, to out-rule if it was cancer or anything else, which I was worried about.

[Interviewer] so did you ever discuss with the GP that you were concerned about that it might be cancer?

I did, yes, speak to the GP about it and, when I said I do belong to [private health insurance firm], so she wrote a referral letter [...] I suggested, myself, please may I have an MRI scan, because X-rays can only pick up so many things, where an MRI can up a lot more, and a lot more detailed. So, yeah, I'm very glad I had it done; very glad.

\section{GP 2 (female)}

I did, so when I requested the bloods, I requested a bone profile as well and full blood count to make sure there was no anaemia. So yes, I did. But I didn't feel, based on her history, that was likely to be cancer. I did the tests to make sure we weren't missing it, but because she was otherwise well it didn't fit with myeloma or anything like that. I still did all the investigations.

[...] Yes, I think the fact that she was running a business and she looks after the grandchildren and she's standing a lot all made me think well this is most likely getting to be mechanical back pain rather than something more sinister. So, I guess from the history what I was gathering was pointing me toward benign

Similarly, Patient 2 was not aware of the diagnostic management strategy that GP 1 was using to resolve her cough (Box 3). She was concerned that the GP did not appear aware of her medical history of whooping cough and silent reflux, and was not aware of the GP's strategy in using a 'trial-of'-treatment' approach (silent reflux) as well as waiting for potential parapertussis symptoms to resolve. The GP, on the other hand, thought that he had clarified this, and that they were working to an 8-week timeframe for reconsultation. In her second interview the patient reflected on her experience and explained that she felt 
that the GP had most likely reached the correct diagnosis, but that the diagnostic approach and next steps had been unclear to her throughout.

Box 3: Misunderstanding diagnostic strategy (Patient 2 and GP 1)

\begin{tabular}{|c|c|}
\hline Patient 2 (female, 58 years old) & GP 1 (male) \\
\hline $\begin{array}{l}\text { How are you feeling at the } \\
\text { moment about the diagnosis? }\end{array}$ & $\begin{array}{l}\text { o, there are viruses called parapertussis viruses that are very like } \\
\text { hooping cough viruses that are continually circulating in the } \\
\text { ommunity and they frequently, not infrequently, can cause }\end{array}$ \\
\hline $\begin{array}{l}\text { R: I'm a bit confused how they } \\
\text { are linked. Whether it is two } \\
\text { separate conditions or...Some of } \\
\text { the symptoms are...are similar. } \\
\text { Yeah. }\end{array}$ & $\begin{array}{l}\text { persistent coughs with paroxysms of coughs where you cough, } \\
\text { cough, cough and can't stop yourself coughing and they last } \\
\text { ages. They last sort of three months and so with her I think with } \\
\text { an eight-week history that was paroxysm and to me it was } \\
\text { probably that or it's silent reflux, so I've given her treatment for } \\
\text { silent reflux and I'll see her again. To be honest if it's }\end{array}$ \\
\hline $\begin{array}{l}\text { Do you feel that the consultation, } \\
\text { at the end, was it clearly linked } \\
\text { to an action? Did you } \\
\text { understand what was going to }\end{array}$ & $\begin{array}{l}\text { parapertussis it would have cleared up by the time I see her again } \\
\text { anyway so if it's cleared up I'll just stop the PPI. If it's not cleared } \\
\text { up I'm guessing I'm probably going to be looking to refer her, } \\
\text { probably just a respiratory referral. }\end{array}$ \\
\hline
\end{tabular}
happen next?

R: Not really, because there was no suggestions for what I could do in the next sort of three or four weeks which would take me up to the end of the three-month period other than increasing the medication for the reflux, but not really anything about the whooping cough.

So, it wasn't clear to you what you should do if anything changed?

R: If I needed to rest or if I needed to drink different amounts. If maybe he'd known a little bit more about the acid reflux and other symptoms. That must've been somewhere back on my records because I went through quite a lot of testing and they found that I had a hernia, and nothing was sinister then. So, it's just at the back of my mind. As he said, I can't be absolutely certain. I know they do normally say that.

These findings indicate that safety netting is dependent on patient understanding of the diagnostic process in addition to comprehension of specific signs, symptoms and timeframes that should trigger 
later actions.

\section{Discussion}

\section{Summary}

This is the first study to our knowledge to examine how patients understand and interpret safety netting advice around potential low-risk lung cancer symptoms. Our results suggest that patients strongly prefer active safety netting, as part of a thorough and logical approach to managing diagnostic uncertainty. Conversely, passive safety netting may be perceived as dismissive and cause patients to delay reconsultation. In contrast, the GPs in our study worried that active strategies, particularly mentioning red flag symptoms, may cause unnecessary concern. Our analysis also suggests that GPs do not always make their safety netting strategy understood, potentially resulting in a misalignment where the GP thinks they have made an active safety netting plan while the patient feels worried or dissatisfied by what they perceive to be a passive safety netting approach. Telephone consultations and the diagnostic overshadowing of COVID-19 on respiratory symptoms were likely to affect GPs' safety netting strategies and patients' appetite for active follow up measures.

\section{Strengths and limitations}

The strength of this study is the focus on patient interpretation of real safety netting experiences, and pairing these with their GPs' aims and perspectives. We interviewed patients from a wide range of geographic areas, giving us a varied picture of patients at risk of lung cancer. Recruitment of GP-patient dyads was limited by the pandemic. The 15 patient participants recruited during the pandemic described significantly different, COVID-related, experiences from patients interviewed pre-pandemic. As much of the focus was on COVID-19, it was challenging to disentangle which actions were 'routine' safety netting and which were pandemic-related. However, given the likely persistence of remote consultation in primary care, our findings will have relevance as new guidance and local practices emerge.

\section{Comparison with existing literature}

Our study builds on previous suggestions from a study of hypothetical safety netting preferences, which reported that patients need active reassurance around reconsultation(11). Furthermore, our study demonstrates that patients are more likely to feel a sense of subjective 'safety' when safety netting is part of a robust and logical consultation, and re-accessing care is assured. This is in line with previous studies reporting patient perceptions of under-support and over-reassurance following all-clear diagnoses(16), and studies showing that patients' perceptions of safety are associated with holistic and individualized care, and challenged by system barriers to healthcare access(20). 
Our study extends recent research looking at the mismatch between safety netting in guidance and practice(1), by collecting empirical data about real consultations rather than hypothetical or consensusdriven designs. Our findings mirror previous interview studies with GPs highlighting variability and uncertainty in safety netting approaches, and worry about managing cancer risk amidst busy workloads $(5,18)$.

\section{Implications for research and/or practice}

Future safety netting research should measure patient understanding and reconsultation behaviour, developing strategies that improve these outcomes without raising unnecessary anxiety. Future studies should conceptualise safety netting as a complex intervention for patient safety and diagnostic management, with the aim to achieve alignment between patient and GP about the presenting problem's significance and next steps. Taking a Health Literacy Universal Precautions approach(21,22), will improve the likelihood that patients understand advice and create an aligned diagnostic strategy(23).

Practices should improve clinical safety netting practice by measuring processes such as delays to reconsultation, missed tests, unfulfilled prescriptions and other measures of concordance. Practices should move away from measuring safety netting quality in terms of missed or delayed cancer diagnosis, which is a relative rarity and a poor 'calibration' measure(24).

\section{Conclusion}

Diagnostic management of patients with low-risk lung cancer symptoms in primary care is a crucial mediator in promoting early diagnosis. Safety netting guidelines do not yet offer solutions that have been proven to promote symptom vigilance and timely reconsultation. Patients prefer active safety netting coupled with thorough consultation techniques and a comprehensible diagnostic strategy, and may respond adversely to passive safety netting advice.

\section{Declarations}

\section{Ethics approval and consent to participate}

The study was approved by the NRES Ethics Committee London Central (REC Ref: 18/LO/1550). We confirm that all participants declared their informed consent to participate in a written form and that all personal identifiers have been removed or disguised so that the persons described are not identifiable and cannot be identified through the details of their stories.

All methods were carried out in accordance with relevant guidelines and regulations.

\section{Consent for publication}

Consent for publication was obtained from all participants. 
Availability of data and materials

The data sets generated and analysed during this study are available from the corresponding author on reasonable request. The approach taken for the study is detailed in the main text and could be reproduced in any similar qualitative interview study.

\section{Competing interests}

The authors have declared no competing interests.

\section{Funding}

This research was funded by a Roy Castle Lung Cancer Foundation research grant (2016/05/Black). The funder was not involved in the design of this study; the collection, analysis, interpretation, or write-up of the results; or in the decision to submit for publication.

This research is linked to the CanTest Collaborative, which is funded by Cancer Research UK [C8640/A23385], of which Fiona Walter and Willie Hamilton are co-Directors, and Cristina Renzi is Principal Clinical Research Fellow.

\section{Authors' contributions}

GB and KW conceived the study in collaboration with all other authors. SvO conducted data collection. $\mathrm{GB}, \mathrm{SvO}$ and $\mathrm{KW}$ undertook the data analysis and interpretation and took the lead in writing the manuscript. All authors contributed to writing, correcting, and approving the final version of the manuscript.

\section{References}

1. Edwards PJ, Ridd MJ, Sanderson E, Barnes RK. Safety netting in routine primary care consultations: an observational study using video-recorded UK consultations. British Journal of General Practice. 2019;69(689):e878-e86.

2. Virgilsen LF, Pedersen AF, Vedsted P, Petersen GS, Jensen H. Alignment between the patient's cancer worry and the GP's cancer suspicion and the association with the interval between first symptom presentation and referral: a cross-sectional study in Denmark. BMC Family Practice. 2021;22(1):129.

3. NICE. Suspected cancer: recognition and referral (NG12). NICE GUIDELINES; 2015.

4. Mendonca SC, Abel G, Saunders C, Wardle J, Lyratzopoulos G. Pre-referral general practitioner consultations and subsequent experience of cancer care: evidence from the English Cancer Patient Experience Survey. European journal of cancer care. 2016;25(3):478-90.

5. Evans J, Macartney JI, Bankhead C, Albury C, Jones D, Ziebland S, et al. How do GPs and patients share the responsibility for cancer safety netting follow-up actions? A qualitative interview study of GPs and patients in Oxfordshire, UK. BMJ open. 2019;9(9):e029316. 
6. Swann R, McPhail S, Witt J, Shand B, Abel GA, Hiom S, et al. Diagnosing cancer in primary care: results from the National Cancer Diagnosis Audit. British Journal of General Practice. 2018;68(666):e63-e72.

7. Redaniel MT, Martin RM, Ridd MJ, Wade J, Jeffreys M. Diagnostic intervals and its association with breast, prostate, lung and colorectal cancer survival in England: historical cohort study using the Clinical Practice Research Datalink. PloS one. 2015;10(5):e0126608.

8. Hamilton W, Peters TJ, Round A, Sharp D. What are the clinical features of lung cancer before the diagnosis is made? A population based case-control study. Thorax. 2005;60(12):1059-65.

9. Almond S, Mant D, Thompson M. Diagnostic safety-netting. British Journal of General Practice. 2009;59(568):872-4.

10. Nicholson BD, Mant D, Bankhead C. Can safety-netting improve cancer detection in patients with vague symptoms? Bmj. 2016;355.

11. Heyhoe J, Reynolds C, Lawton R. The early diagnosis of cancer in primary care: A qualitative exploration of the patient's role and acceptable safety-netting strategies. European journal of cancer care. 2020;29(1):e13195.

12. Lyratzopoulos $G$, Vedsted $P$, Singh H. Understanding missed opportunities for more timely diagnosis of cancer in symptomatic patients after presentation. British journal of cancer. 2015;112(1):S84-S91.

13. Bjerager $M$, Palshof $T$, Dahl R, Vedsted $P$, Olesen F. Delay in diagnosis of lung cancer in general practice. British Journal of General Practice. 2006;56(532):863-8.

14. Black G, Sheringham J, Spencer-Hughes V, Ridge M, Lyons M, Williams C, et al. Patients' Experiences of Cancer Diagnosis as a Result of an Emergency Presentation: A Qualitative Study. PLOS ONE. 2015;10(8):e0135027.

15. Birt L, Hall N, Emery J, Banks J, Mills K, Johnson M, et al. Responding to symptoms suggestive of lung cancer: a qualitative interview study. BMJ open respiratory research. 2014;1 (1):e000067.

16. Renzi C, Whitaker KL, Winstanley K, Cromme S, Wardle J. Unintended consequences of an 'allclear'diagnosis for potential cancer symptoms: a nested qualitative interview study with primary care patients. British Journal of General Practice. 2016;66(644):e158-e70.

17. Bankhead C, Heneghan C, Hewitson P, Thompson M. Safety netting to improve early cancer diagnosis in primary care: development of consensus guidelines. University of Oxford: Department of Primary Health Care. 2011.

18. Tompson A, Nicholson BD, Ziebland S, Evans J, Bankhead C. Quality improvements of safety-netting guidelines for cancer in UK primary care: insights from a qualitative interview study of GPs. British Journal of General Practice. 2019;69(689):e819-e26.

19. Heyhoe J, Reynolds C, Dunning A, Johnson O, Howat A, Lawton R. Patient involvement in diagnosing cancer in primary care: a systematic review of current interventions. British Journal of General Practice. 2018;68(668):e211-e24.

20. Rhodes P, Campbell S, Sanders C. Trust, temporality and systems: how do patients understand patient safety in primary care? A qualitative study. Health Expect. 2016;19(2):253-63. 
21. Brega A, Barnard J, Mabachi N, Weiss B, DeWalt D, Brach C, et al. AHRQ Health Literacy Universal Precautions Toolkit, Second Edition. In: Colorado Health Outcomes Program University of Colorado Anschutz Medical Campus, editor. Second Edition ed. Rockville, MD: Agency for Healthcare Research and Quality; 2015.

22. Zabar S, Hanley K, Wilhite JA, Altshuler L, Kalet A, Gillespie C. In the room where it happens: do physicians need feedback on their real-world communication skills? : BMJ Publishing Group Ltd; 2020.

23. Amelung D, Whitaker KL, Lennard D, Ogden M, Sheringham J, Zhou Y, et al. Influence of doctorpatient conversations on behaviours of patients presenting to primary care with new or persistent symptoms: a video observation study. BMJ quality \& safety. 2020;29(3):198-208.

24. Omron R, Kotwal S, Garibaldi BT, Newman-Toker DE. The diagnostic performance feedback "calibration gap": why clinical experience alone is not enough to prevent serious diagnostic errors. AEM education and training. 2018;2(4):339-42.

\section{Supplementary Files}

This is a list of supplementary files associated with this preprint. Click to download.

- APPENDIX1BlackvanOs.docx 